\title{
AUTO-IMMUNE RESPONSE TO NUCLEUS PULPOSUS IN THE RABBIT
}

\author{
W. P. Bobechko, Toronto, Canada, and Carl Hirsch, Gothenburg, Sweden \\ From the Department of Orthopaedics, University of Gothenburg
}

Intervertebral disc structures undergoing degenerative changes often induce secondary reactions characterised by hypervascularisation in the surrounding connective tissues. These degenerative changes produce brief clinical symptoms and often are poorly correlated to the pathological state. Although clinical symptoms subside, more widespread degenerative changes become manifest in segments of the spine.

In recent years, with the increased emphasis on auto-immune mechanisms (Rebuck 1960), it has been suggested that the pathogenesis of chronic low back pain may be part of such a process (Naylor 1962). This becomes more plausible when one considers the peculiar make-up of that mass of totally avascular mucoprotein, the nucleus pulposus.

The nucleus pulposus is normally contained tightly within the annulus, and after its embryological formation it no longer normally makes any vascular contact with the systemic circulation (Hirsch and Schajowicz 1952). This situation is analogous to at least two other human situations in which derangement is known to produce an auto-immune response. The enclosed thyroglobulin in Hashimoto's disease of the thyroid and the similarly enclosed vitreous humour of the eye in the production of sympathetic ophthalmia are two such autoimmune diseases. In both cases when the avascular protein is exposed to the systemic circulation the protein is auto-antigenic and is rejected by a lymphocytic auto-immune reaction. More recently an auto-immune reaction has been postulated in idiopathic Addison's disease with lymphocytic destruction of the adrenal gland (Steiner, Langer, Schatz and Volpe 1960).

At the present time emphasis has been focused on regional lymph nodes as the site of antibody production in many immunological responses (Scothorne and McGregor 1955, Burwell 1964). This antibody does not enter the systemic circulation to give humoral antibody titres but remains fixed within the cytoplasm of certain lymphocytes in the "cell-bound antibody" state (Turk and Stone 1964). In this cell-bound state the antibody can be carried from the site of production within the primary regional node by efferent lymphatics to the site of the antigen (or auto-antigen) and here produce a chronic inflammatory reaction by interaction of antigen and antibody (Burwell 1964).

The cells of the lymphocyte series which produce the antibody in the regional nodes and hold it in their cytoplasm as a ribonucleic acid are variously termed immunoblasts, haemocytoblasts, large lymphoid cells or pyroninophilic cells (Horowitz, Bauer, Paronetto, Abrams, Watkins and Popper 1964). This cell-bound antibody can be detected in lymph nodes in a non-specific manner by the use of a methyl-green pyronin stain. This stains the antibody in the cytoplasm a distinct vivid red and is termed pyroninophilia (Burwell 1964). Inactive lymph nodes contain only few such pyronin-positive cells.

A new hypothesis based on this type of auto-immune response has been formulated as a possible explanation for the perplexing cases of chronic inflammatory reaction so often encountered as nerve root adhesions during a "negative disc exploration" in patients with chronic low back pain (Hirsch and Schajowicz 1952). This concept (Fig. 1) depends on whether auto-antibodies can in fact develop to one's own nucleus pulposus if this is exposed to a blood supply.

This paper presents the results of a study in which an attempt was made to induce the production of auto-immune antibodies in the primary regional auricular lymph nodes in the 
rabbit to the animal's own nucleus pulposus (Fig. 1, phase 1). The nucleus pulposus was removed from its avascular site within the intact annulus and implanted in a vascular site in the ear, where it was exposed to the systemic circulation. Primary and secondary draining lymph nodes and similar control nodes from the other side were then examined to see if the nucleus pulposus would be rejected as "not self" by the production of auto-immune and cell-bound antibodies as if to a foreign antigen. It has been shown by Burwell and Gowland (1961) that a small piece of autogenous iliac bone similarly implanted in the rabbit's ear produces virtually no immune reaction. Their work thus serves as a further control for our experiments.

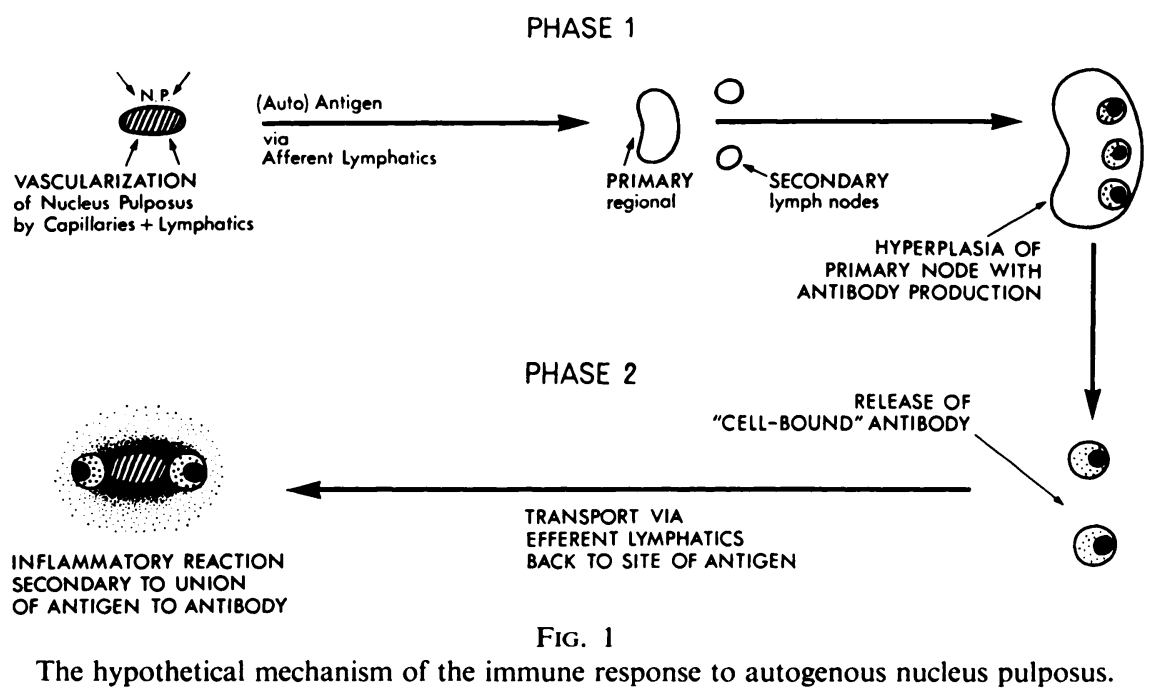

\section{METHODS AND MATERIALS}

Adult rabbits clear of obvious infection and weighing about two kilograms were used. Skin flaps were raised on the dorsal surface of both ears near the base by a technique like that of Burwell and Gowland (1961). The underlying flap of perichondrium was stripped off the ear cartilage and a small circular pocket four millimetres in diameter was made in the cartilage to serve as a receptacle for the nucleus pulposus material. The nucleus pulposus was thus enclosed between the dorsal and ventral layers of perichondrium. The lumbar disc space was then approached by a ventral route and the semi-solid nucleus pulposus removed and placed into the right ear pocket. Both ear flaps and abdominal incision were closed with silk suture. The left ear served as control since no disc material was implanted there.

A total of twenty-one animals were killed at intervals of from three days to six weeks, usually two animals at a time (Figs. 2 and 3). At the intervals of six days and four weeks, when the greatest responses were expected from homograft studies, the group consisted of three animals.

At death, parts of both ear sites were fixed in Zenker's fixative and lead acetate. Histological sections were made and stained with haematoxylin and eosin, Dominici and toluidine blue.

The constant primary lymph node at the base of each ear was dissected out; its size and consistency were recorded, and then half of the node was fixed in Zenker's fluid for haematoxylin and eosin sections and the remaining half in Carnoy's fixative. The secondary lymph nodes in either side of the neck were also dissected out and similarly fixed. The portions of the lymph nodes fixed in Carnoy's fixative were stained by the Unna-Pappenheim methylgreen method for pyronin. 


\section{OBSERVATIONS}

The healing of the ear flaps proceeded at the expected rate and normally except that during the first week more oedema was consistently present about the right ear flap containing the insert of nucleus pulposus than about the left, control flap.

At death the dissected lymph nodes at the ear bases and along the carotid chains were measured. Throughout the entire six weeks, and especially after twelve days and after six

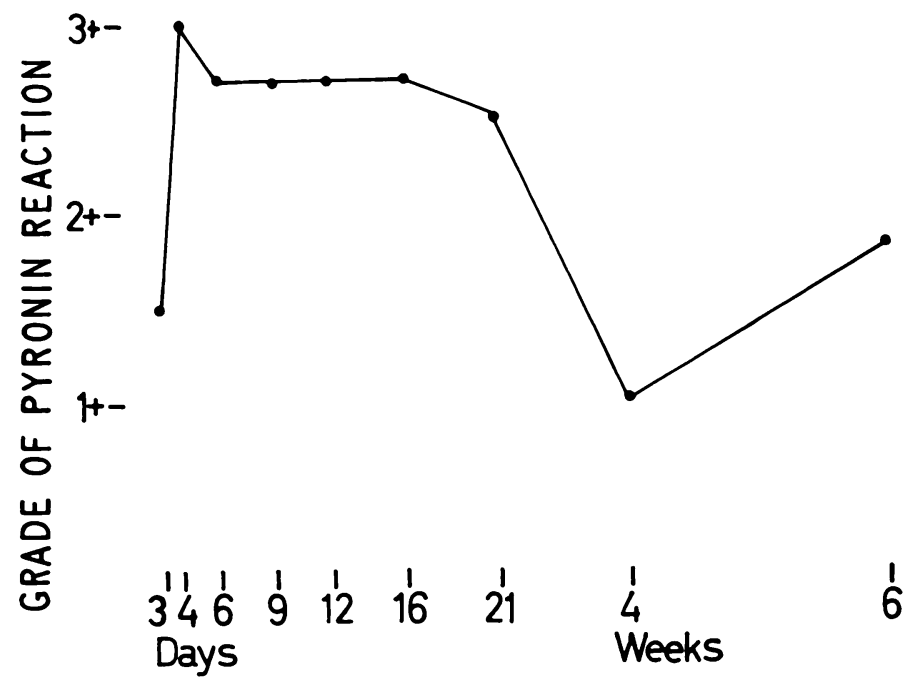

FiG. 2

Antibody response to nucleus pulposus in right primary lymph node.

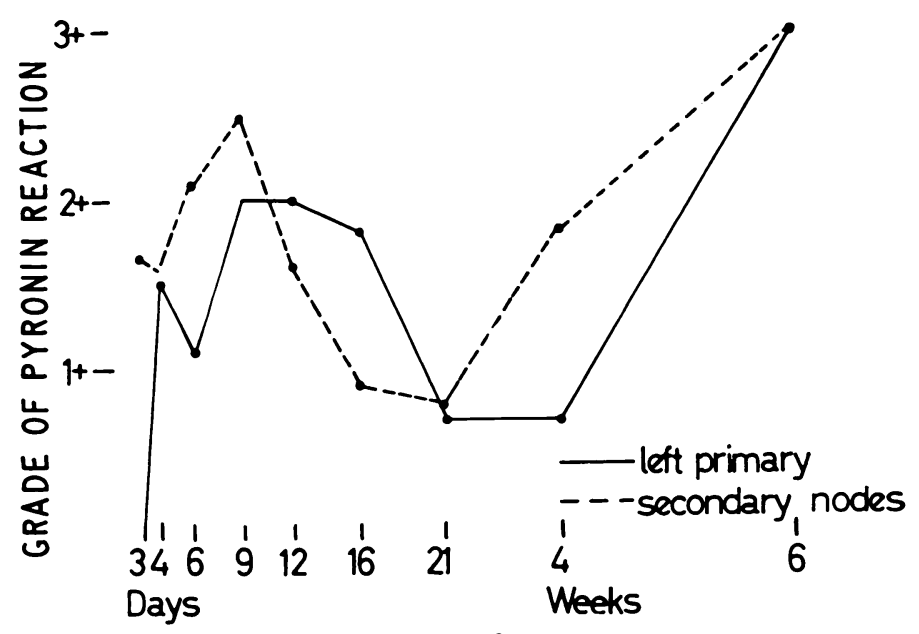

FIG. 3

Antibody response in control (left primary) and in secondary lymph nodes.

weeks, the right primary node was invariably larger (up to 1.5 centimetres long, or twice the size of the left, control side). The primary lymph node on the left (control) side, however, in most cases was also slightly enlarged as compared to a completely normal lymph node at this very specific site in the intact animal.

The secondary nodes showed no recognisable enlargement on either side within the first three weeks.

Histology-No histological changes that were specifically different from the normal inflammatory reaction and that could be readily graded were noted at either of the ear sites. 
The primary and secondary lymph nodes on both sides were graded $(0$ to $3-)$ for the large lymphoid cells which showed a red pyronin reaction in the cytoplasm after staining by the Unna-Pappenheim method. This grading ( 0 to $3+)$ was based upon observation of lymph nodes under high power $(\times 500)$ magnification. At the zero grade only infrequent pyroninpositive cells were present. The $3+$ grade exhibited multiple areas with a greater than 75 per cent concentration of distinctly pyronin-positive large lymphoid cells within each high power field. These are the specific cells usually located towards the cortices of the node and probably indicative of antibody synthesis (Burwell and Gowland 1961) (Fig. 4).

In addition it was seen that the lymphoid tissue fixed in Carnoy's fluid (non-aqueous) showed a marked natural fluorescence in the blue light range (Zeiss fluorescent microscope, Osram mercury vapour lamp with B.G.12 energising filter and 50/44 barrier filters). This distinct yellow fluorescence was limited to the cytoplasm (but only in cells which had a

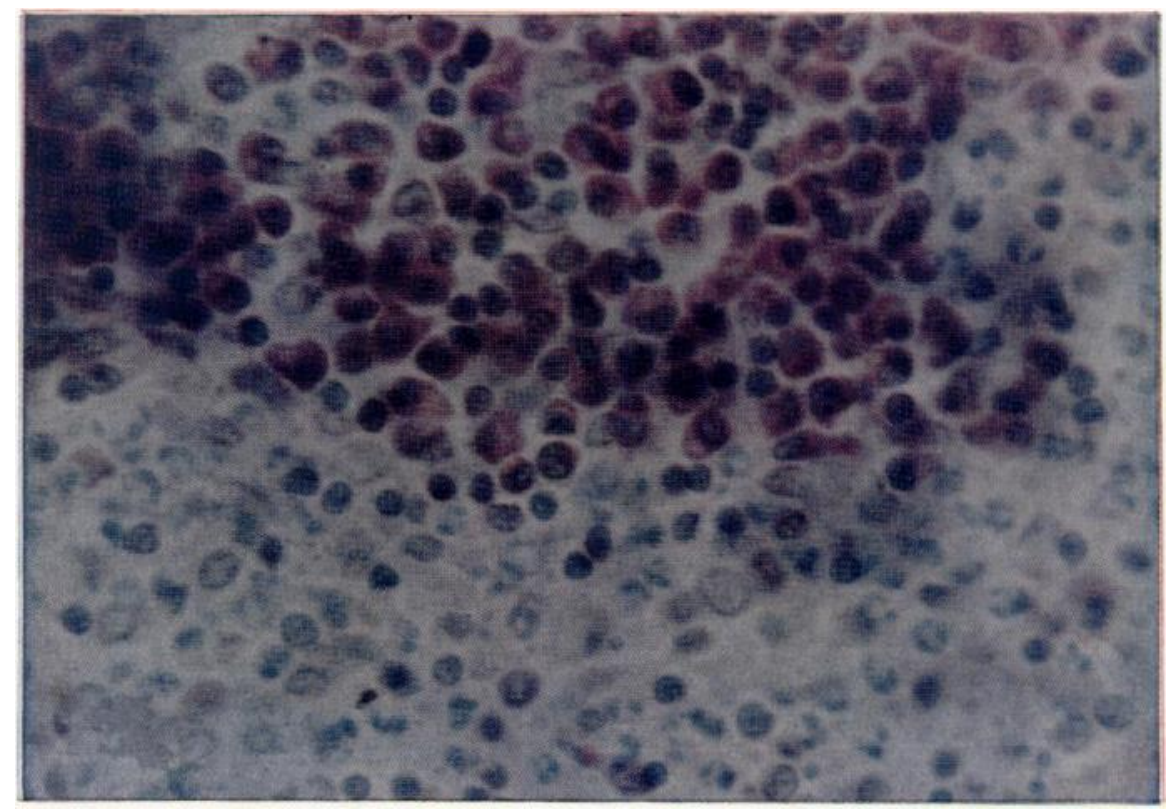

FIG. 4

Histological appearance. Primary lymph node draining site of implantation. Pyronin stain. Marked pyroninophilia in cytoplasm of large lymphoid cells in upper part of field. Many small lymphocytes in lower part. ( $\times 200$.)

pyronin-positive cytoplasm) and provided an additional method for assessing the antibody production of these specific lymphoid cells (Figs. 5 and 6). Lymphoid cells without the red pyroninophilia did not exhibit the yellow cytoplasmic fluorescence. Furthermore, unstained sections fixed in Carnoy's fluid and sections fixed in formalin but stained for pyronins did not show this fluorescence.

With both of these methods (degree of pyroninophilia and cytoplasmic fluorescence) all lymph nodes were graded ( 0 to $3+$ ) for antibody production. The examiner had no previous knowledge of the side of origin of the lymph node. The results are expressed in the graphs in Figures 2 and 3. These show that there is a more rapid, more intense and prolonged antibody response in the right primary lymph node draining the nucleus pulposus insert. This reaction is greatest at four days but remains strong for three weeks (Fig. 2).

An antibody type reaction does, however, occur in the left control primary lymph node and in both sets of secondary nodes (Fig. 3). When plotted (Fig. 3) the reactions in these three control nodes are graphically similar but are most active at a different time (nine to 


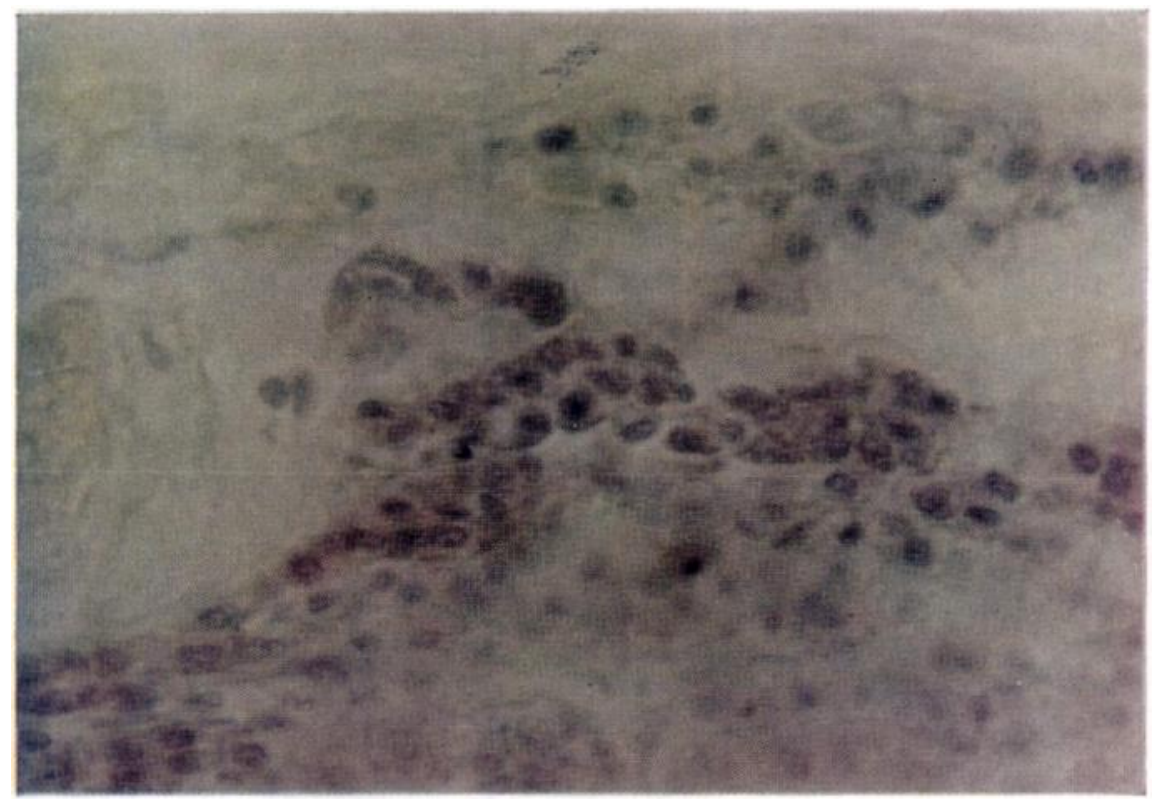

FiG. 5

Primary lymph node draining site of implantation. Pyronin stain. Note pyroninophilia in large lymphoid cells. $(200$.

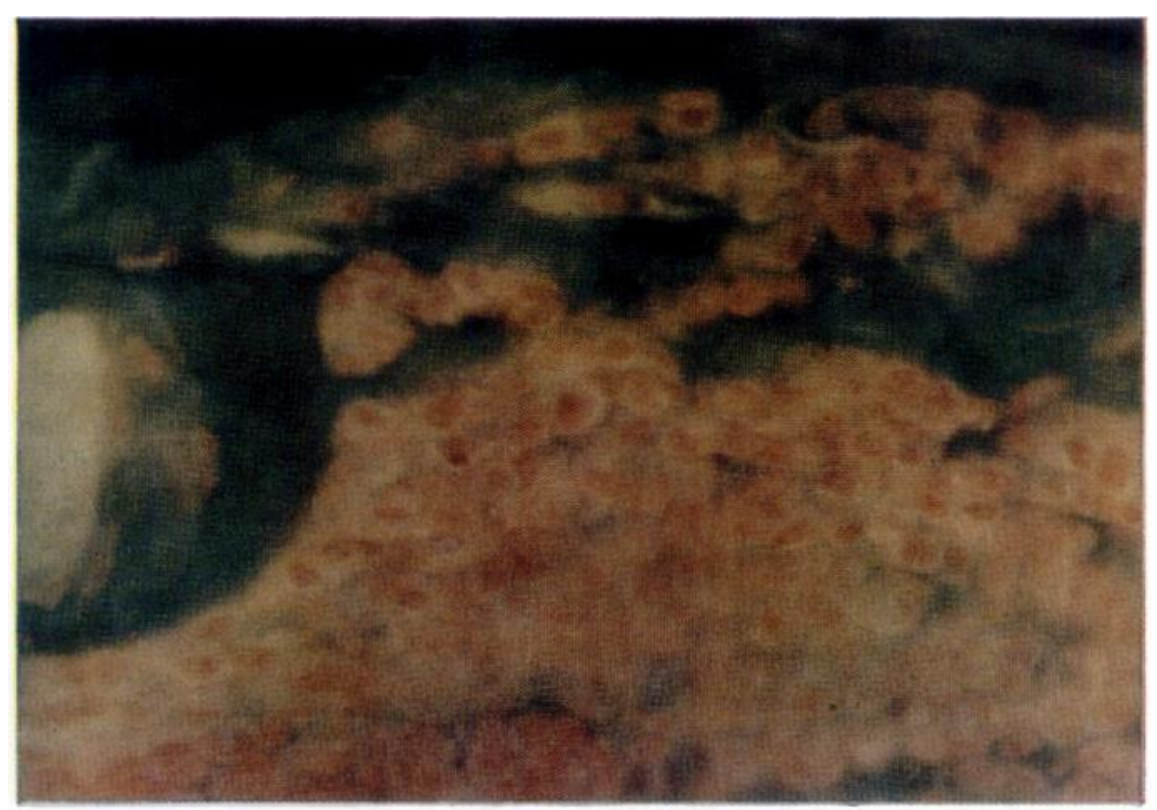

FIG. 6

The same field as in Figure 5, viewed with blue light. Pyronin stain. The nuclei of the large lymphoid cells appear orange, whereas the cytoplasm shows yellow fluorescence. $(\times 200$.) 
twelve days). There is less pyroninophilia than in the right primary node and fluorescence is briefer. These three control nodes thus present a common pattern of response differing from that in the right primary node.

Statistical analysis of results-The distribution of the findings (up to twenty-one days) shown in the groups (Figs. 2 and 3) is summarised in Table I. There were insufficient samples at respective points on the curves for a valid comparison at each day. However, the differences in distribution of the grading for all groups in the first twenty-one days are significant.*

There are no significant differences between the control and nucleus pulposus nodes after three weeks. It is of interest that all lymph nodes undergo a marked secondary response at six weeks.

\section{DISCUSSION}

These experimental findings in the rabbit suggest that autogenous nucleus pulposus material can excite an auto-antibody response in the regional lymph nodes. This response is distinctly different from the reaction of the control and secondary lymph nodes. This interpretation is, however, dependent on two rather non-specific reactions. The first is that of the pyronin-staining reaction within the cytoplasm of large lymphoid cells. This staining

TABLE I

Distribution of Pyroninophilia and Fluorescence in Primary Nodes Draining Nucleus Pulposus and in Control Nodes

\begin{tabular}{|c|c|c|c|c|}
\hline \multirow{2}{*}{ Grade of reaction } & \multicolumn{2}{|c|}{ Nucleus pulposus nodes } & \multicolumn{2}{|c|}{ Control nodes } \\
\hline & Number & Percentage & Number & Percentage \\
\hline 0 & 3 & $7 \cdot 5$ & 16 & $13 \cdot 8$ \\
\hline $1-$ & 5 & $12 \cdot 5$ & 34 & $29 \cdot 3$ \\
\hline $2-$ & 11 & $27 \cdot 5$ & 40 & $34 \cdot 4$ \\
\hline $3-$ & 21 & $52 \cdot 5$ & 26 & $22 \cdot 4$ \\
\hline Number of samples & 40 & & 116 & \\
\hline
\end{tabular}

reaction is believed to represent antibody synthesis in these cells within the primary draining lymph node (Turk and Stone 1964). The second basis for interpretation is an unproven and non-specific natural fluorescence. However, similar methods have been used by Sainte-Marie and Coons (1964). This yellow fluorescence is a more distinct and possibly more sensitive indicator of an alteration occurring in the cytoplasm of those lymphoid cells (if fixed in Carnoy's fluid) which also show a positive pyroninophilia (Sedlin 1964).

The auto-immune antibody response within the primary lymph node reaches a rapid maximum at four days but then, unlike most homograft reactions which are short lived (Burwell and Gowland 1961), remains at a high level for three weeks. The significance of this prolonged response is uncertain. In addition a secondary rise in cell-bound antibody appears in all lymph nodes at six weeks as shown by strong pyroninophilia and fluorescence. This may be part of a generalised systemic antibody response but no check was made for a humeral antibody titre.

Further support for this hypothesis has been provided on two occasions when an anterior lumbar intercorporeal fusion (for failed posterior disc surgery) was being performed. There was a marked hyperplasia and enlargement of the regional para-aortic lymph nodes about the lumbar spine. Initially it was thought that this lymph node enlargement represented an

* The 5 per cent level of probability is accepted as statistically significant in this study. 
underlying infection. On culture and section of the nodes no bacteria were found. Unfortunately, at that time the lymphatic tissue was not fixed in Carnoy's fixative to permit examination for pyroninophilia. With the increase in the number of anterior lumbar intercorporeal fusions performed it would be of interest to sample these readily obtainable para-spinal lymph nodes, fix them in Carnoy's fixative and examine them for antibody production.

At the present state of our knowledge of auto-immunity it appears that the first phase of the auto-immune process suggested here (Fig. 1) does occur. One finds enlargement of lymph nodes along with microscopic changes compatible with auto-immunisation.

\section{SUMMARY AND CONCLUSIONS}

1. A hypothesis outlining an auto-immune mechanism for antibody production against autogenous nucleus pulposus is presented.

2. Auto-antibodies to autogenous nucleus pulposus have been experimentally produced in rabbits.

3. These antibodies are cell-bound within lymphoid cells and are greatest in primary lymph nodes. This antibody is demonstrated by a positive pyronin reaction.

4. Lymphoid cells fixed in Carnoy's fluid and stained for pyronins also show a distinct natural yellow fluorescence. This fluorescence occurs only in the cytoplasm of those cells which are pyronin-positive and presumably producing antibody.

5. The lymph node phase of the reaction is greatest at four days and is sustained for three weeks. A secondary generalised lymph node response occurs in all lymph nodes at six weeks.

Grateful acknowledgment is given for the helpful advice and use of the laboratory facilities of Dr P. I. Brånemark, Laboratory of Experimental Biology, Department of Anatomy, University of Gothenburg, and of the advice given by Professor G. Asboe-Hansen of the Rigs Hospital, Copenhagen, in the interpretation of the histological material.

This work, supported by a grant from the Swedish State Medical Research Council, was completed in Sweden during the tenure by one of us (W. P. B.) of a McLaughlin Travelling Fellowship from the University of Toronto.

\section{REFERENCES}

BURwell, R. G. (1964): Biological Mechanisms in Foreign Bone Transplantation. Immunological Mechanisms. In Modern Trends in Orthopaedics. Edited by J. M. P. Clark. Vol. 4, p. 138. London: Butterworths.

Burwell, R. G., and Gowland, G. (1961): Studies in the Transplantation of Bone. II. The Changes Occurring in the Lymphoid Tissue after Homografts and Autografts of Fresh Cancellous Bone. Journal of Bone and Joint Surgery, 43-B, 820.

HiRsCH, C., and SCHAJOwiCz, F. (1952): Studies on Structural Changes in the Lumbar Annulus Fibrosus. Acta Orthopaedica Scandinavica, 22, 184.

Horowitz, R. E., Bauer, H., Paronetto, F., Abrams, G. D., Watkins, K. C., and Popper, H. (1964): The Response of the Lymphatic Tissue to Bacterial Antigen. American Journal of Pathology, 44, 747.

NAylor, A. (1962): The Biophysical and Biochemical Aspects of Intervertebral Disc Herniation and Degeneration. Annals of the Royal College of Surgeons of England, 31, 91.

Rebuck, J. W., ed. (1960): The Lymphocyte and Lymphocytic Tissue. New York: Paul Hoeber Inc.

Sainte-Marie, G., and Coons, A. H. (1964): Studies on Antibody Production. Journal of Experimental Medicine, 119, 743.

Scothorne, R. J., and McGregor, I. A. (1955): Cellular Changes in Lymph Nodes and Spleen Following Skin Homografting in the Rabbit. Journal of Anatomy (London), 89, 283.

Sedlin, E. D. (1964): Personal communication.

Steiner, J. W., Langer, B., Schatz, D. L., and VolPe, R. (1960): Experimental Immunologic Adrenal Injury. Journal of Experimental Medicine, 112, 187.

Turk, J. L., and Stone, S. H. (1964): Implications of the Cellular Changes in Lymph Nodes during the Development and Inhibition of Delayed Type Hypersensitivity. In Cell-bound Antibodies, a symposium edited by D. B. Amos and H. Koprowski. P. 51. Philadelphia: The Wistar Institute Press. 